\title{
Collapsing dynamics of attractive Bose-Einstein condensates
}

\author{
L. Bergé ${ }^{1}$ and J. Juul Rasmussen ${ }^{2}$ \\ ${ }^{1}$ Commissariat à l'Energie Atomique, CEA/DAM - Ile de France, B.P. 12, 91680 Bruyères-le-Châtel, France. \\ ${ }^{2}$ Risø National Laboratory, Optics and Fluids Dynamics Department, P.O. Box 49, 4000 Roskilde, Denmark.
}

(October 31, 2018)

\begin{abstract}
The self-similar collapse of $3 \mathrm{D}$ and quasi-2D atom condensates with negative scattering length is examined. 3D condensates are shown to blow up following the scenario of weak collapse: The inner core of the condensate diverges with an almost zero particle number, while its tail distribution spreads out to large distances with a constant density profile. For this case, the 3-body recombination arrests the collapse, but it weakly dissipates the atoms. The confining trap then reforms the condensate at later times. In contrast, 2D condensates undergo a strong collapse: The atoms stay mainly located at center and recombination sequentially absorbs a significant amount of particles.
\end{abstract}

PACS numbers : 03.75.Fi, 32.80.Pj, 42.65.Jx.

A few years ago, Bose-Einstein condensates (BECs) were discovered in trapped clouds of alkali atoms [1,2]. Among those, ${ }^{7} \mathrm{Li}$ atoms are known to be characterized by attractive interactions with a negative scattering length, $a_{0}<0$ [2]. This promotes a collapse-type instability, which yields a singular increase in the BEC wavefunction. It occurs when the number of particles, $\tilde{N}$, exceeds a threshold value, $\tilde{N}_{c} \sim l_{0} /\left|a_{0}\right|$, where $l_{0}=(\hbar / m \omega)^{1 / 2}$ is the amplitude of zero point oscillations in the confining trap of frequency $\omega$ and $m$ is the atom mass. BECs formed in ${ }^{7} \mathrm{Li}$ gas develop several sequences of collapse [3]. From the initial cooling, the condensate is first fed by the thermal cloud of uncondensed atoms. Then, once the number of atoms is above $\tilde{N}_{c}$, the condensate sharply concentrates with an increasing density. As the density rises, inelastic collisions as 3-body molecular recombination increase, which arrests the collapse. The thermal cloud is next believed to re-fill in the condensate, that reaches $\tilde{N}>\tilde{N}_{c}$ and then collapses again. The cycle of collapses repeats many times until the gas comes to equilibrium. This scenario is supported by numerical integrations of the Gross-Pitaevskii (G.-P.) equation [4, 5]:

$$
i \partial_{t} \psi=-\Delta_{r} \psi+r^{2} \psi-|\psi|^{2} \psi-i \eta|\psi|^{m} \psi+i \gamma \psi,
$$

where $r=\tilde{r} / l_{0}, t=\tilde{t} \omega / 2, \quad \psi=\tilde{\psi}\left(8 \pi l_{0}^{2}\left|a_{0}\right|\right)^{1 / 2}$ are the dimensionless time, coordinates and wavefunction of the BEC (tilde refers to physical quantities). Here, the loss/gain mechanisms are described by the last two terms of Eq. (1), where $\eta \ll 1$ and $\gamma \ll 1$ are the coefficients related to the local recombinational decrease of the condensate density and to the flux of particles from the nonequilibrium thermal cloud to the condensate, respectively. At leading order, we consider 3-body recombinations with $m=4$. The normalized number of particles, $N=\int|\psi|^{2} d \vec{r}$, is related to its physical counterpart as $\tilde{N}=N\left(l_{0} / 8 \pi\left|a_{0}\right|\right) \simeq 86.7 N$. It was recently shown that stationary condensates defined by $\psi_{s}(r, t)=$ $\chi(r, \mu) \mathrm{e}^{-i \mu t}$ with chemical potential $\mu$ are stable whenever they satisfy $d N_{e q} / d \mu<0$, where $N_{e q}(\mu)=\int|\chi|^{2} d \vec{r}$
[6]. Along the curve $N_{e q}(\mu), 3 \mathrm{D}$ condensates then requires $N_{e q} \leq N_{c}=14.45$ for being stable. In physical units, this threshold yields the precise critical number for stability: $\tilde{N}_{c}=1252$ atoms, for the ${ }^{7} \mathrm{Li}$ parameters $a_{0}=-1.45 \mathrm{~nm}$ and $l_{0}=3.16 \mu \mathrm{m}$ used in [2, 3[.5].

Numerical simulations [4] of Eq. (11) revealed that, near the collapse moment, the condensate expands with a density profile exhibiting a low-amplitude, almost flat plateau, $|\psi|^{2} r^{2} \rightarrow$ const, from $r>1$. This plateau-like behavior was again numerically found in [5], where the free collapse was described as a "black hole". Following this scenario, the increase in the BEC density is fueled by particles drawn from throughout the outer region $(r>1)$, while the density outside form imploding ripples. However, it was suggested that the asymptotic plateau in $r^{2}|\psi|^{2}$ may not be constant, but instead diverges in time. Thus, the singular dynamics of $3 \mathrm{D}$ collapsing BECs is still questionable and a self-consistent model for BEC collapse is actually missing. Describing the structure of such collapses is of utmost importance, in order to understand the influence of recombination, trap confinement and re-feeding over several collapse events.

In this letter, we examine the self-similar nature of collapsing BECs. For 3D isotropic condensates the number of particles is analytically shown to vanish near center and, outside, a constant plateau in the density profile, $r^{2}|\psi|^{2}$, is actually formed. From this dynamics, 3-body recombination arrests the collapse by removing a limited number of particles from the condensate. We also briefly investigate 2D condensates. As justified in [7], quasi2D BECs can be produced from 3D atom clouds with a density frozen on a Gaussian shape $\propto \mathrm{e}^{-\tilde{z}^{2} / l_{0}^{2}}$, when the particles are tightly confined along the longitudinal axis. In what follows, $2 \mathrm{D}$ and $3 \mathrm{D}$ condensates are considered as isotropic, radially-symmetric objects, since the self-compression induced by the collapse dynamics make them have comparable sizes along each direction.

Let us first discuss the inertial regime of collapse, for which we set $\gamma=\eta=0$ in Eq. (11). Collapse oc- 
curs in the sense that the mean-square width of attractive BECs, $\left\langle r^{2}\right\rangle=N^{-1} \int r^{2}|\psi|^{2} d \vec{r}$, tends to zero in finite time (see, e.g., Pitaevskii [8]). From the inequality $N \leq\left\langle r^{2}\right\rangle \int|\nabla \psi|^{2} d \vec{r}$,9], the vanishing of $\left\langle r^{2}\right\rangle$ leads to the blow-up of the gradient norm, which in turn implies the divergence of the integral $\int|\psi|^{4} d \vec{r}$ in the conserved Hamiltonian of Eq. (11):

$$
H=\int|\nabla \psi|^{2} d \vec{r}-\frac{1}{2} \int|\psi|^{4} d \vec{r}+N\left\langle r^{2}\right\rangle .
$$

By virtue of the mean-value theorem $|\psi|^{4} \leq$ $\max _{r}|\psi|^{2} \int|\psi|^{2} d \vec{r}$, the maximum amplitude of the wavefunction also blows up in finite time. It should be emphasized that the blow-up generally occurs before $\left\langle r^{2}\right\rangle$ reaches zero [9]. To examine the shape of collapsing condensates, we introduce the self-similarlike substitution:

$$
\psi(\vec{r}, t)=a^{-\alpha}(t) \phi(\vec{\xi}, \tau) \mathrm{e}^{i \lambda \tau-i \beta \xi^{2} / 4},
$$

where $\vec{\xi}=\vec{r} / a(t), \tau(t) \equiv \int_{0}^{t} d u / a^{2}(u)$ and $\beta=-a \dot{a}$ (dot means differentiation with respect to time). Here, the parameter $\lambda \sim-\mu$ must be positive in the absence of the trap for making $\phi$ localized. The function $a(t)$ represents the BEC scale length that vanishes as collapse develops, and $\phi(\vec{\xi}, \tau)$ is a regular function with amplitude of order unity. As $a(t) \rightarrow 0$, it is assumed that $\phi$ converges to an exactly self-similar form $\phi(\vec{\xi})$, which no longer depends explicitly on time, i.e., $\partial_{\tau} \phi \rightarrow 0$. In this limit, the right balance between the two integrals in Eq. (2) requires $\alpha=1$, in order to assure the finiteness of $H$. The particle number $N=\int|\psi|^{2} d \vec{r}$ then reads $N=a^{D-2}(t) \int|\phi|^{2} d \vec{\xi}$ and the dynamics drastically changes following the space dimension number $D$. Setting thus $\alpha=1$ and plugging Eq. (3) into (1) transforms the G.-P. equation into

$$
\begin{gathered}
i \partial_{\tau} \phi+\Delta_{\xi} \phi+|\phi|^{2} \phi+\epsilon\left[\xi^{2}-\xi_{T}^{2}\right] \phi-a^{4} \xi^{2} \phi+ \\
i \eta a^{2-m}|\phi|^{m} \phi-i \gamma a^{2} \phi=0
\end{gathered}
$$

where $\Delta_{\xi}=\xi^{1-D} \partial_{\xi} \xi^{D-1} \partial_{\xi}, \epsilon \equiv-\frac{1}{4} a^{3} \ddot{a}=\frac{1}{4}\left(\beta^{2}+\beta_{\tau}\right)$ and $\xi_{T}^{2} \equiv \epsilon^{-1}[\lambda+i \beta(D / 2-1)]$ is viewed as a turning point. After an initial stage during which the trap gathers the particles at center, the wavefunction diverges freely on short time scales $\Delta \tilde{t} \ll \omega^{-1}$. It becomes hyperlocalized at $r \sim 0$ with $a(t) \rightarrow 0$, so that the effect of trapping can be neglected. Near the collapse point, refeeding from the surrounding cloud is also inefficient. Moreover, 3body recombination does not act, as long as the BEC radius satisfies $\eta a^{2-m}|\phi|^{m} \ll|\phi|^{2}$. In this regime, Eq. (4) thus reduces to the self-similarly transformed nonlinear Schrödinger (NLS) equation, whose properties, accurately verified in [9 11], are recalled below.

In the self-similar limit $a(t) \rightarrow 0, \partial_{\tau} \phi \rightarrow 0$, the timedependent function $\epsilon$ converges to $\beta^{2} / 4$, which, for selfconsistency, converges to a constant, $\epsilon_{0}$. (ii) The solution $\phi$ in Eq. (4) can be decomposed into a core contribution $\phi_{c}$ extending in the range $\xi<\xi_{T}$, i.e., $r<r_{T} \equiv a(t)\left|\xi_{T}\right|$, and a tail $\phi_{T}$ defined in the complementary spatial domain $\xi>\xi_{T}$, i.e., $r>r_{T}$. (iii) The self-similar assumption $\partial_{\tau} \phi \rightarrow 0$ holds provided that $\xi<\xi_{\max } \equiv A\left|\xi_{T}\right| / a(t)$, where $A=$ const $\gg 1$. Selfsimilar solutions are thus limited by the boundary radius $r_{\max }=A\left|\xi_{T}\right| \gg 1$. Knowing this, the solution $\phi$ decomposes as $\phi=\left.\phi_{c}\right|_{\xi<\xi_{T}}+\left.\phi_{T}\right|_{\xi_{T}<\xi<\xi_{\max }}$. In the long distance domain, the nonlinearity vanishes, so that $\phi_{T}$ can be determined from the linear version of Eq. (4) by means of WKB methods 97. As a result, the wavefunction $\psi$ reads near the collapse point $\left(\epsilon=\beta^{2} / 4\right)$ as

$$
\begin{gathered}
\psi(\vec{r}, t)=\frac{\mathrm{e}^{i \lambda \int_{0}^{t} \frac{d u}{a^{2}(u)}}}{a(t)} \times \\
\left\{\left.\phi_{c}\left(\frac{r}{a}, \epsilon\right) \mathrm{e}^{-i \beta r^{2} / 4 a^{2}}\right|_{0 \leq r<r_{T}}+\left.\frac{C(\beta)}{(r / a)^{1+i \lambda / \beta_{0}}}\right|_{r_{T}<r<r_{\text {max }}}\right\}, \\
|C(\beta)|^{2} \simeq \frac{2 \phi^{2}(0)}{\beta\left|\xi_{T}\right|^{D-2}} \mathrm{e}^{f(\lambda / \beta)}, \\
\left.\begin{array}{l}
\text { The scaling law } a(t) \text { in the inertial range of collapse is } \\
\beta
\end{array}\right)-\frac{\pi \lambda}{\beta}+\left(\frac{D}{2}-1\right)(1+2 \ln 2)-\frac{\lambda}{\beta} \arctan \left[\frac{\beta}{\lambda}\left(\frac{D}{2}-1\right)\right] . \\
\text { then identified through the continuity equation for } \psi \text { : } \\
\int_{0}^{r_{\text {max }}} \partial_{t}|\psi|^{2} r^{D-1} d r=-\left.2 r^{D-1}|\psi|^{2} \partial_{r} \arg (\psi)\right|^{r_{\text {max }}} \\
-2 \eta \int_{0}^{r_{\text {max }}}|\psi|^{m+2} r^{D-1} d r+2 \gamma \int_{0}^{r_{\text {max }}}|\psi|^{2} r^{D-1} d r, \quad(7)
\end{gathered}
$$

where $\arg [\psi(r=0)]=0$. By applying the solution (5), (6) to Eq. (7) and Taylor-expanding $\phi_{c}$ around $\epsilon=\beta^{2} / 4=\epsilon_{0}$, the contraction scale $a(t)$ is indeed readily determined for $\eta=\gamma=0$ from the dynamical system:

$$
c_{1} \beta_{\tau} \simeq-\frac{c_{2}}{\beta} \mathrm{e}^{f(\lambda / \beta)}+D-2,
$$

where $\left.c_{1} \propto \operatorname{Re} \int \phi_{0}^{*} \partial_{\epsilon} \phi\right|_{\epsilon_{0}} d \vec{\xi}$ and $c_{2}>0$ are constants.

We specify the structure of the collapse for $3 \mathrm{D}$ and quasi-2D BECs separately:

1 - Three-dimensional condensates: Let us first describe the inertial range of collapse. For $D=3$, Eq. (8) shows that $\beta$ rapidly attains a fixed point $\beta_{0}>0$ corresponding to the self-similar state $\beta_{\tau}=0$. The scaling law $a(t) \sim\left(t_{c}-t\right)^{1 / 2}$ follows, where $t_{c}$ denotes the collapse moment. The characteristics of a $3 \mathrm{D}$ collapse is that the number of particles is not preserved self-similarly in the whole spatial domain, since $N=a(t) N\{\phi\}$ with $N\{\phi\}=\int|\phi|^{2} d \vec{\xi}$. By virtue of Eq. (5), we see that, near the collapse instant, $N$ behaves as 
$N \simeq N_{\text {core }}(t)+N_{\text {tail }}(t)=a(t)\left[N\left\{\phi_{c}\right\}(t)+N\left\{\phi_{T}\right\}(t)\right]$, where

$$
\begin{gathered}
N\left\{\phi_{c}\right\}=4 \pi \int_{0}^{\xi_{T}}\left|\phi_{c}\right|^{2} \xi^{2} d \xi \simeq O(1) \\
N\left\{\phi_{T}\right\}=4 \pi|C(\beta)|^{2} \int_{\xi_{T}}^{\xi_{\text {max }}} d \xi \simeq 4 \pi|C(\beta)|^{2} r_{\max } / a(t),
\end{gathered}
$$

so that almost all $N$ lies in the tail, $N_{\text {tail }}(t)=$ $4 \pi\left|C\left(\beta_{0}\right)\right|^{2} r_{\text {max }}$, as $a(t) \rightarrow 0$. Therefore, a 3D collapse takes place at the center of the trap where the wavefunction $|\psi|=|\phi| / a(t)$ diverges. However, it is accompanied by an expulsion of particles towards the largedistance domain $r \gg r_{T}(t)$. Thus, after the onset of collapse, the singularity develops, not by taking particles from outside as in the "black hole" scenario proposed in [5], but by ejecting particles outward the core domain. This is a weak collapse, as originally defined in Ref. 12] for the free NLS equation. The solution $\psi$ blows up at center, near which the number of particles becomes zero, i.e., $N_{\text {core }}(t)=4 \pi \int_{0}^{r_{T}(t)}\left|\psi_{\text {core }}\right|^{2} r^{2} d r \rightarrow 0$, as $r_{T}(t) \sim a(t)$ vanishes. Accordingly, the tail of the BEC wavefunction in the outer domain extends in space with the time-independent density: $r^{2}|\psi|^{2} \rightarrow\left|C\left(\beta_{0}\right)\right|^{2}$ $=$ const, deduced from Eq. (5). This constant depends on the values of $\beta_{0}$ and $\left|\xi_{T}\right|$. For the scaling law $a(t)=a_{0}\left(t_{c}-t\right)^{1 / 2}$ with $a_{0}$ set equal to the unity without loss of generality, $\beta_{0}=1 / 2$ and numerical integrations of the 3D NLS equation with no trap [11] emphasize the values $\phi_{0} \equiv \phi(0)=1.39$ and $\lambda=0.545$, which yield $\left|\xi_{T}\right| \sim 3.1$ and $\left|C\left(\beta_{0}\right)\right|^{2} \simeq 0.17 \sim 0.2$. A tail contribution $r^{2}|\psi|^{2} /|\psi(t=0)|^{2}$ about 0.2 in magnitude for $r \geq 1$ seems compatible with the density profiles computed in Refs. [4, [5] in the vicinity of the collapse moment. The formation of stationary plateau-like density profiles was numerically confirmed in 10,11] by means of very fine numerical schemes that solved the rescaled equation (4) with high accuracy and could access huge growths in $|\psi|^{2}$. In contrast, the same plateau was claimed to diverge in time by only refining the spatial grid in [5]. We suspect that numerical computations in [5] suffered serious lack of resolution, which prevented the authors from concluding correctly on the constancy of $r^{2}|\psi|^{2}$ at large distances.

We now discuss the dissipative/gain regime, that involves collisional losses and feeding by the thermal cloud. The influence of these two effects on the condensate particle number is described by the last two terms in the continuity equation (7). By introducing the self-similar shape (5), these terms are found to read $-2 \eta a^{1-m} \int_{0}^{\xi_{T}}\left|\phi_{c}\right|^{m+2} \xi^{2} d \xi$ and $2 \gamma a \int_{\xi_{T}}^{\xi_{\max }}\left|\phi_{T}\right|^{2} \xi^{2} d \xi$ for $a(t) \ll 1$, respectively. Hence, 3-body recombination mainly acts on the core part of the solution $\left|\psi_{c}\right|=$ $\left|\phi_{c}\right| / a(t)$, whereas re-feeding by the surrounding cloud is efficient in the outer region, where the main amount of particles is residing, with $2 \gamma \int_{0}^{r_{\max }}|\psi|^{2} r^{2} d r \simeq 2 \gamma N_{\text {tail }}(t)$.
Collisions begin to be active when $a(t)$ decreases so much that $\eta a^{2-m}|\phi|^{m} \simeq|\phi|^{2}$ [Eq. (四], i.e., this contribution saturates the blow-up induced by the cubic nonlinearity, for $m>2$. By means of this relation, the number of particles $\Delta N_{\text {loss }}$ lost from the condensate during one collapse event is then yielded by that in the core region, $N_{\text {core }}$. Inelastic collisions are thus able to stop the collapse, but they cannot remove a lot of particles, the major part of $N \simeq N_{\text {tail }}$ starting to be transferred to large distances via the weak collapse. Explicitly, we find by using Eq. (5) expressed with arbitrary $m$ and $D$ :

$$
\Delta N_{\text {loss }} \simeq-2^{D-1}\left(\phi_{0}\left|\xi_{T}\right|\right)^{D} \pi \eta^{(D-2) /(m-2)} / D .
$$

For $D=3$ and $m=4$, the particle loss is estimated as $\Delta N_{\text {loss }} \simeq 4 \pi \sqrt{\eta} \phi_{0}^{3} \xi_{T}^{3} / 3=0.5$, when we choose the value $\eta=2.2 \times 10^{-6}$ physically justified in [5] and employ $\phi_{0}=1.39$ and $\xi_{T}=3.1$. Thus, in physical units, the number of atoms removed during a single blow-up event is $\Delta \tilde{N}_{\text {loss }}=44$ atoms. This loss of particles agrees with recent numerical observations [13]. Here, approximately 300 particles were lost from a condensate of initial number $N_{0}=1260$ atoms, in a sequence of 5-6 individual blow-up events with peak amplitudes $|\psi|_{\max } \sim \eta^{1 / 2-m}$. As discovered by Vlasov et al. [14] and confirmed in [11], an untrapped, 3D collapsing field is damped within a very short time window, in which the field experiences secondary blow-up events of duration $\sim \eta^{2 / m-2}$, as long as nonlinear dissipation remains active. These arise while most of the atoms form a broad disribution surrounding the collapsing core and are directed outwards. Collapse is not arrested abruptly, but it is distributed through a series of individual spikes. This "distributed collapse" [14] resembles the "intermittent implosions" numerically detected in [13]. Note that these intermittent implosions, which overlap as one collapse sequence over a trap period, may not be resolved in real experiments. Their integrated effect should only yield a smooth decrease in $\tilde{N}$.

Once collapse is limited, it is necessary to understand the mechanism underlying reformation and periodic resurgences of spiky amplitudes in the condensate [3]. We emphasize that after the collapse stage most particles lie in the outer domain $1 \leq r<r_{\max }$, where the atoms feel both the trap curvature and re-feeding by the thermal cloud. The latter re-injects particles into the $\mathrm{BEC}$ according to $N(t) \simeq N_{t a i l} \mathrm{e}^{2 \gamma t}$, i.e., on time scales $t \simeq(2 \gamma)^{-1} \ln \left(1+\Delta N / N_{0}\right)$. For reasonable values of $\gamma=3 \times 10^{-3}$ [5], the physical time for refilling the condensate with $\Delta N / N_{0} \approx 0.25$ is too long for justifying the spiky oscillations occurring periodically at times $\sim \omega^{-1}$, mentioned in, e.g., [13. We have thus to analyze the stage of trap reconfinement at earlier instants for which refeeding is negligible. To this aim, we define the centroid of the expanded structure by $\vec{X}(t) \equiv N^{-1} \int \vec{r}|\psi|^{2} d \vec{r}$. From algebraic manipulations of Eq. (11) with $\gamma=\eta=0$, this centroid is found to obey 
the relation $\ddot{\vec{X}}+4 \vec{X}=\overrightarrow{0}$. Simple phase transformation [15] keeps equation (11) unchanged when passing over to the frame moving with $X(t)$. It is then easy to conclude that, from a reference coordinate $\vec{X}\left(t_{0}\right) \neq \overrightarrow{0}$ that locates the BEC expanded in the outer domain at a given time $t_{0}$, the condensate will return to the center of the trap over physical times $=\pi / 2 \omega \sim \omega^{-1}$ after the collapse moment. Once reconfined with $N<N_{c}$, the condensate is close to an equilibrium, that is described by the ground state solution $\psi_{s}=\chi(r, \mu) \mathrm{e}^{-i \mu t}$ of the G.-P. equation. As shown in Ref. [6] (see also [16]), ground states are stable with $d N_{e q} / d \mu<0$ for rather broad condensates $(0.72<\mu<3)$. They are unstable and collapse with $d N_{e q} / d \mu>0$ for rather narrow condensates $(\mu<0.72)$. In this range, the threshold $N_{c}>N_{e q}$ decreases with $|\mu|$ as $N_{c}(\mu) \leq 18.94 /|\mu|$. It is thus highy problable that under the strong compression induced by the trap, the equilibrium state confined at center corresponds to a narrow, unstable $\psi_{s}$ undergoing a new collapse sequence with a lower critical number of particles. This may repeat over several trap periods, until the refeeding becomes a keyplayer for making the resulting BEC bifurcate to a stable equilibrium reached at a weak number of atoms [3].

2- Quasi-two-dimensional condensates: The dynamics significantly changes when one considers quasi-2D BECs. For $\eta=\gamma=0$, the scaling law $a(t)$ inferred from Eq. (8) with $D=2$ indeed behaves with a twice-logarithmic correction: $a(t) \simeq a_{0} \sqrt{t_{c}-t} / \sqrt{\ln \ln \left[1 /\left(t_{c}-t\right)\right]}$ [9-11]. Since $\beta \simeq \pi / \ln \tau(t) \rightarrow 0$ as $\tau(t) \simeq \ln \left[1 / t_{c}-t\right] \rightarrow+\infty$, the exponential contribution in the tail amplitude of Eq. (6) decreases to zero, while the core boundary $\xi_{T}=\sqrt{\lambda / \epsilon}$ increases slowly to infinity when $t \rightarrow t_{c}$. As a result, collapse takes place with a core solution $\phi_{c}$ providing the principal contribution in the wavefunction $\psi . N$ is mainly given by $\int\left|\phi_{c}\right|^{2} d \vec{\xi}$, which relaxes to the critical value $N_{c}=\int|\chi|^{2} d \vec{\xi} \simeq 11.7$. Below this number, stationary condensates are stable [6]. So, most of particles stay located around the center of the trap, when collapse develops. This scenario meets the definition of the so-called strong collapse, which contrasts with the $3 \mathrm{D}$ weak collapse that promotes a leak of atoms to large distances. The relative variations in the particle number due to collisional losses are governed by the estimate $\Delta N_{\text {loss }} \simeq-2 \phi_{0}^{-2} \eta a^{2-m} \int\left|\phi_{c}\right|^{m+2} d \vec{\xi}$. As $a(t)$ decreases, the part burnt by recombination is here estimated by $\Delta N_{\text {loss }} / N \simeq-2 /(1+m / 2)$, when we apply the evaluation $\eta a^{2-m} \approx|\phi|^{2-m}$ and model $\phi$ around a Gaussian with intensity $I_{0}: \phi=\sqrt{I_{0}} \mathrm{e}^{-\xi^{2} / 2}$. For $m=4$, 3-body recombination then removes about more than one-half of the condensed atoms. Although rough, this estimate does not explicitly vary with the coefficient $\eta$, as already indicated by Eq. (10), and it slightly decreases with $m$, in agreement with the numerical observations of Ref. [11]. Collapse is thus arrested by 3-body recombinations that sequentially burn a substantial amount of atoms per col- lapse event. This agrees with the numerical results of Refs. 111,14, from which $\Delta N_{\text {loss }}$ is about $0.25-0.3 \times N_{c}$.

In conclusion, we have shown that BECs with attractive interactions collapse with a mean radius contracting like $\sqrt{t_{c}-t}$ at leading order, where $t_{c}$ denotes the collapse moment. In 3D, the collapse is weak: the amplitude of the wavefunction blows up at center, while particles are expelled outwards with a constant density profile $r^{2}|\psi|^{2} \rightarrow$ const. Recombination losses limit the collapse, but they only damp a few percents of the atoms at each blow-up. Several blow-up events develop within one collapse sequence. The condensate is then reconfined by the trap and can further undergo more collapse cycles, as long as a stable state has not been reached. Quasi-2D condensates are subject to a strong collapse, in the sense that the number of particles remains mostly confined at center. In this case, recombination removes a significant fraction (up to 0.5) of particles per collapse event.

This work was partly supported by the Danish Natural Sciences Foundation (snf-grant 9903273).

[1] M.H. Anderson et al., Science 269, 198 (1995); K.B. Davis et al., Phys. Rev. Lett. 75, 3969 (1995).

[2] C.C. Bradley, C.A. Sackett, J.J. Tollett, and R.G. Hulet, Phys. Rev. Lett. 75, 1687 (1995). C.C. Bradley, C.A. Sackett, and R.G. Hulet, Phys. Rev. Lett. 78, 985 (1997).

[3] C.A. Sackett, J.M. Gerton, M. Welling, and R.G. Hulet, Phys. Rev. Lett. 82, 876 (1999).

[4] Yu. Kagan, A.E. Muryshev, and G.V. Shlyapnikov, Phys. Rev. Lett. 81, 933 (1998).

[5] A. Eleftheriou and K. Huang, Phys. Rev. A 61, 043601 (2000); See also M. Ueda and K. Huang, Phys. Rev. A 60, 3317 (1999).

[6] L. Bergé, T.J. Alexander, and Yu.S. Kivshar, Phys. Rev. A 62, 023607 (2000).

[7] H. Gauck et al., Phys. Rev. Lett. 81, 5298 (1998); A.I. Safonov et al., Phys. Rev. Lett. 81, 4545 (1998). See also D.S. Petrov, M. Holzmann, and G.V. Shlyapnikov, Phys. Rev. Lett. 84, 2551 (2000) and references therein.

[8] L.P. Pitaevskii, Phys. Lett. A 221, 14 (1996).

[9] L. Bergé, Phys. Rep. 303259 (1998); J. Juul Rasmussen and K. Rypdal, Phys. Scr. 33, 481 (1986).

[10] D.W. McLaughlin et al., Phys. Rev. A 34, 1200 (1986); B.J. LeMesurier et al., Physica D 31, 78 (1988); 32, 210 (1988).

[11] N.E. Kosmatov, V.F. Shvets, and V.E. Zakharov, Physica D 52, 16 (1991).

[12] V.E. Zakharov and E.A. Kuznetsov, Sov. Phys. JETP 64, 773 (1986).

[13] H. Saito and M. Ueda, arXiv:cond-mat/0002393 (2000).

[14] S.N. Vlasov, L.V. Piskunova, and V.I. Talanov, Sov. Phys. JETP 68, 1125 (1989).

[15] L. Bergé, Phys. Plasmas 4, 1227 (1997).

[16] C. Huepe, S. Métens, G. Dewel, P. Borckmans, and M.E. Brachet, Phys. Rev. Lett. 82, 1616 (1999). 